Gisele Lacerda Chave Vieira ${ }^{\mathrm{a}}$

${ }^{a}$ Fundação Hospitalar do Estado de Minas Gerais. Belo Horizonte, MG, Brasil.

Contato:

Gisele Lacerda Chave Vieira

E-mail:

giselelacerdavi@gmail.com

A autora declara que a pesquisa não recebeu financiamento e que não há conflitos de interesses.

A autora informa que o trabalho não é baseado em dissertação ou tese e que não foi apresentado em eventos científicos.

\section{Agressão física contra técnicos de enfermagem em hospitais psiquiátricos}

\author{
Physical aggression against nursing technicians \\ in psychiatric hospitals
}

\section{Resumo}

Objetivo: caracterizar os casos de agressão física contra técnicos de enfermagem em dois hospitais psiquiátricos e analisar os fatores relacionados à ocorrência desses eventos. Métodos: estudo transversal realizado com 125 técnicos de enfermagem. Foi utilizado questionário autoaplicável e pesquisa documental. Foram analisados os registros de ocorrências de agressões físicas junto ao serviço de saúde e segurança do trabalho dos hospitais. Foram calculadas frequências, médias, desvios-padrões e percentis. A associação entre a ocorrência de agressão física e as variáveis pesquisadas foi verificada por meio de regressão logística. Resultados: 96 (76,8\%) participantes relataram ter passado pela experiência de agressão física. Entre estes, 94,3\% referiram consequências psicológicas, 28,4\% relataram lesão física. Apenas 30,3\% dos respondentes afirmaram ter recebido suporte da instituição após a ocorrência. Foi identificada associação estatística significante entre agressão física e percepção de insegurança, sexo feminino e maior tempo de trabalho em instituições psiquiátricas. Também foi observado subnotificação de casos. Conclusão: a agressão física é vivenciada com alta frequência entre os técnicos de enfermagem, que relatam pouco suporte institucional e sentimentos de insegurança no ambiente de trabalho, mostrando a necessidade de instituir medidas que melhorem as condições de trabalho e previnam a violência.

Palavras-chave: violência no trabalho; enfermagem psiquiátrica; saúde do trabalhador; agressão; hospitais psiquiátricos.

\begin{abstract}
Objective: to characterize cases of physical aggression against nursing technicians in two psychiatric hospitals and to analyze the factors related to the occurrence of these events. Methods: cross-sectional study, using self-administered questionnaire and documentary research involving 125 nursing technicians. We analyzed records of physical aggression occurrences from the occupational health and safety units of the hospitals and calculated variables frequencies, averages, standard deviation, and percentiles. The association between the occurrence of physical aggression and the variables studied was verified using logistic regression. Results: 96 (76.8\%) participants reported having experienced physical aggression. Among those, 94.3\% referenced psychological consequences and 28.4\% reported physical injuries. Only $30.3 \%$ of the participants stated to have received support from the hospital after the occurrence. There was a significant statistic association between physical aggression and perception of insecurity, female gender, and longer time working in psychiatric institutions. Underreporting of cases was also observed. Conclusion: physical assault is experienced with high frequency among nursing technicians, who report little institutional support and feelings of insecurity in the workplace, showing the need to introduce measures to improve working conditions and prevent violence.
\end{abstract}

Keywords: violence at work; psychiatric nursing; occupational health;

aggression; psychiatric hospitals.
Revisado: 23/10/2016

Aprovado: 04/11/2016 


\section{Introdução}

A violência contra os profissionais que trabalham em instituições de saúde tem se tornado um importante desafio para a saúde ocupacional. Dessa forma, as pesquisas têm modificado, gradualmente, o foco sobre as questões tradicionais e buscado investigar fatores importantes que afetam a qualidade de vida no ambiente de trabalho ${ }^{1}$. Nesse sentido, destaca-se que episódios de violência contra os profissionais da saúde estão relacionados ao desenvolvimento de estresse, ansiedade, exaustão emocional e redução da qualidade de vida no trabalho ${ }^{1-3}$.

No Brasil, estudos foram realizados em unidades de emergência, hospitais gerais e na atenção primária $^{4-6}$. As pesquisas realizadas em hospitais psiquiátricos de outros países apresentam diversos fatores como determinantes da violência nestas instituições, geralmente relacionados ao perfil do paciente, como idade, gênero, presença de sintomas psicóticos e uso de álcool e outras drogas ${ }^{7,8}$.

Os profissionais de enfermagem apresentam as maiores taxas como vítimas de violência física, psicológica, verbal ou sexual quando comparados aos demais profissionais de saúde ${ }^{9}$. Em hospitais psiquiátricos essas taxas são ainda maiores, variando de 1,7\% a $71,6 \%^{10-13}$, indicando que as características dos pacientes ou as relativas aos profissionais de saúde podem ser fatores determinantes para a ocorrência desses eventos ${ }^{7,8,12}$. As taxas de violência física, especificamente, chegam a representar $17 \%$ dos casos. Outros fatores que parecem contribuir para aumentar a chance desses eventos são o tempo de contato com os pacientes, o tempo de experiência profissional e o dimensionamento de recursos humanos ${ }^{12,14,15}$.

Entre os profissionais de enfermagem ${ }^{16,17}$, os técnicos (nível médio de escolaridade) são os que mais se encontram em contato direto com os pacientes nas instituições de saúde. Sendo assim, são os mais expostos aos riscos de agressão física. Destaca-se que, no Brasil, esse fenômeno ainda é visto com certa naturalidade, na medida em que é reconhecido como inerente ao exercício do trabalho, contribuindo para a subnotificação dos episódios de violência ${ }^{5}$.

Considerando a escassez de estudos no Brasil ${ }^{18}$ que tratem dessa questão em hospitais psiquiátricos, este trabalho possui o objetivo de caracterizar os casos de agressão física contra técnicos de enfermagem e analisar os fatores relacionados à ocorrência destes eventos em dois hospitais psiquiátricos de Minas Gerais. A hipótese principal desta pesquisa é que o maior tempo de experiência profissional e ser do sexo masculino são fatores que reduzem a chance de os técnicos de enfermagem passarem por experiências de agressão física.

\section{Metodologia}

Trata-se de estudo transversal realizado em dezembro de 2014 em dois hospitais psiquiátricos da rede pública de Belo Horizonte, Minas Gerais, Brasil. As instituições somam 253 leitos e 255 técnicos de enfermagem. Esses hospitais oferecem serviços de atendimento ambulatorial, urgência e internação hospitalar.

Todos os profissionais de enfermagem de nível médio de escolaridade foram convidados a participar. Os critérios de inclusão foram: atuar como técnico de enfermagem no hospital e prestar assistência direta aos pacientes. Os enfermeiros não foram incluídos por dois motivos: ausência de relatos de agressão física contra esta categoria profissional no período estudado e por apresentarem menor tempo de contato com os pacientes internados quando comparados aos técnicos de enfermagem.

Foram seguidos todos os preceitos éticos, de acordo com a Resolução $n^{\circ} 466 / 2012$, tendo aprovação no ano de 2013 pelo CEP da Fundação Hospitalar do Estado de Minas Gerais com Parecer $\mathrm{n}^{\mathrm{o}}$ 237.265. Os participantes assinaram o Termo de Consentimento Livre e Esclarecido.

A coleta de dados foi realizada em duas etapas. A primeira etapa consistiu no uso de questionário autoaplicável com questões relacionadas às características sociodemográficas, à experiência profissional e à ocorrência de agressão física, sendo: idade em anos completos, sexo do profissional, tempo de vínculo com a instituição em anos completos, tempo de trabalho em instituições psiquiátricas (em anos completos), carga horária de trabalho, número de vínculos empregatícios, turno de trabalho, percepção de segurança no ambiente de trabalho, experiência de agressão física no ambiente de trabalho, suporte da instituição à ocorrência do evento, consequências físicas e psicológicas, setor de ocorrência, registro da Comunicação de Acidente de Trabalho (CAT), necessidade de afastamento do serviço. Já na segunda etapa, realizada por meio de pesquisa documental, foram analisadas as CATs e os dados registrados referentes à ocorrência de agressão física contra os profissionais da instituição no último ano, sendo: turno em que o evento ocorreu, setor, sexo do paciente e do profissional envolvido, diagnóstico do paciente à internação e seu histórico de dependência química, tempo de internação em dias e características da agressão física.

Os dados foram duplamente digitados em uma planilha de Excel, sendo analisados por meio do software STATA versão 12.0. A análise descritiva foi realizada calculando frequências e percentuais para as variáveis categóricas; média e desvio-padrão para as variáveis quantitativas. Procedeu-se com 
análise estatística construindo-se modelos de regressão logística univariada. As variáveis com p $<0,20$ foram selecionadas para a análise de regressão logística múltipla. O teste de Hosmer-Lemeshow foi utilizado para analisar o ajuste do modelo. Foi considerado o nível de significância de 5\%. Os valores missing não foram incluídos na análise.

\section{Resultados}

Dos 255 profissionais de enfermagem, 240 atenderam aos critérios de inclusão. Desses, 30 encontravam-se de licença médica, férias regulamentares ou férias-prêmio, 25 se recusaram a participar do estudo e 60 não retornaram os questionários. No total, 125 profissionais responderam ao questionário, sendo 71 técnicos de um hospital e 54 de outro. Os profissionais que não participaram do estudo não apresentaram diferenças em relação às variáveis sexo, idade e tempo de vínculo com o serviço quando comparados aos profissionais que participaram da pesquisa.

Os 125 profissionais que participaram da pesquisa apresentaram média de idade de 43,9 anos (DP $=9,5$ anos), sendo a maior parte do sexo feminino ( $\mathrm{n}=88 ; 70,4 \%)$. A maioria trabalha no período diurno ( $\mathrm{n}=75 ; 60,0 \%$ ), possui carga horária de 40 horas semanais ( $\mathrm{n}=66 ; 52,8 \%$ ) e apenas um vínculo empregatício $(\mathrm{n}=80 ; 64,0 \%)$. No que diz respeito à percepção de segurança em relação a agressões no ambiente de trabalho, dos 120 que responderam a este quesito, $103(85,8 \%)$ relataram não se sentirem seguros (Tabela 1).
Dos 125 participantes, 96 (76,8\%) relataram já ter passado por alguma experiência de agressão física no trabalho. Entre esses, a maior parte dos casos ocorreu com profissionais do sexo feminino $(\mathrm{n}=72$; $75,0 \%$ ) e no período diurno ( $n=55 ; 57,3 \%)$. No que se refere à percepção de segurança dos trabalhadores agredidos, $83(90,2 \%)$ relataram se sentirem inseguros no hospital (Tabela $\mathbf{1}$ ).

A maioria dos profissionais de enfermagem possuía tempo de trabalho abaixo de cinco anos em instituições psiquiátricas ( $\mathrm{n}=86 ; 68,8 \%$ ), assim como em tempo de vínculo com o hospital ( $\mathrm{n}=83$; $66,4 \%$ ). De igual maneira, a maioria dos profissionais que relatou ter sofrido algum tipo de agressão física apresentou tempo de trabalho em instituições psiquiátricas ( $\mathrm{n}=61 ; 63,5 \%$ ) e tempo de vínculo com o hospital ( $\mathrm{n}=61 ; 63,5 \%$ ) inferiores a cinco anos (Tabela 1).

Entretanto, ao se verificar a média do tempo de trabalho em instituições psiquiátricas e a média do tempo de vínculo nos hospitais pesquisados, essas foram maiores entre os profissionais de enfermagem que sofreram algum tipo de agressão física. Entre os profissionais que não sofreram agressão física, o tempo médio de trabalho em instituições psiquiátricas foi de 6,3 anos (DP $=4,1$ anos) e a média do tempo de vínculo dos participantes nas instituições pesquisadas foi de 6,4 anos ( $\mathrm{DP}=2,5$ anos). Já, entre esses profissionais que vivenciaram algum tipo de agressão física, o tempo médio de trabalho em instituições psiquiátricas foi de 7,5 anos (DP $=1,0)$ e 0 tempo médio de vínculo desses profissionais com os hospitais estudados foi de 7,3 anos (DP $=1,0)$.

Tabela 1 Características sociodemográficas e relativas à experiência profissional dos técnicos de enfermagem de duas instituições psiquiátricas, conforme a vivência de agressão física. Belo Horizonte, MG, Brasil, 2014

\begin{tabular}{|c|c|c|c|}
\hline & \multicolumn{3}{|c|}{ Agressão física $-n(\%)$} \\
\hline & Total & Sim & Não \\
\hline \multicolumn{4}{|c|}{$\operatorname{Sexo}(n=125)$} \\
\hline Feminino & $88(70,4)$ & $72(75,0)$ & $16(55,2)$ \\
\hline Masculino & $37(29,6)$ & $24(25,0)$ & $13(44,8)$ \\
\hline \multicolumn{4}{|c|}{ Sente-se seguro no trabalho $(n=120)$} \\
\hline Sim & $17(14,2)$ & $9(9,8)$ & $8(28,6)$ \\
\hline Não & $103(85,8)$ & $83(90,2)$ & $20(71,4)$ \\
\hline \multicolumn{4}{|c|}{ Turno de trabalho $(\mathrm{n}=125)$} \\
\hline Diurno & $75(60,0)$ & $55(57,3)$ & $20(69,0)$ \\
\hline Noturno & $50(40,0)$ & $41(42,7)$ & $9(31,0)$ \\
\hline \multicolumn{4}{|c|}{ Carga horária de trabalho (horas semanais) $(\mathrm{n}=125)$} \\
\hline 20 & $2(1,6)$ & $2(2,1)$ & $0(0,0)$ \\
\hline 30 & $57(45,6)$ & $51(53,7)$ & $6(20,0)$ \\
\hline 40 & $66(52,8)$ & $42(44,2)$ & $24(80,0)$ \\
\hline
\end{tabular}

(Continua) 
Tabela 1 Continuação...

\begin{tabular}{|c|c|c|c|}
\hline & \multicolumn{3}{|c|}{ Agressão física $-n(\%)$} \\
\hline & Total & Sim & Não \\
\hline \multicolumn{4}{|c|}{ Número de empregos $(\mathrm{n}=125)$} \\
\hline 1 & $80(64,0)$ & $59(62,1)$ & $21(70,0)$ \\
\hline 2 & $43(34,4)$ & $35(36,8)$ & $8(26,7)$ \\
\hline 3 & $2(1,6)$ & $1(1,0)$ & $1(3,3)$ \\
\hline \multicolumn{4}{|c|}{ Tempo de trabalho em instituições psiquiátricas (anos) ( $n=125)$} \\
\hline 0 a 5 anos & $86(68,8)$ & $61(63,5)$ & $25(86,2)$ \\
\hline$>5$ anos & $39(31,2)$ & $35(36,5)$ & $4(13,8)$ \\
\hline \multicolumn{4}{|c|}{ Tempo de vínculo na instituição (anos) (n = 125) } \\
\hline 0 a 5 anos & $83(66,4)$ & $61(63,5)$ & $22(75,9)$ \\
\hline$>5$ anos & $42(33,6)$ & $35(36,5)$ & $7(24,1)$ \\
\hline
\end{tabular}

A Tabela 2 apresenta informações relacionadas aos casos de agressões. O número total de respostas variou conforme indicado na tabela. Entre os dados apurados, destaca-se: apenas 30,3\% dos respondentes afirmaram ter recebido suporte da instituição após a ocorrência do episódio de agressão física; 28,4\% disseram ter como consequência algum grau de lesão física; e 94,3\% afirmaram apresentar consequências psicológicas em decorrência da agressão. Com relação ao local de ocorrência dos eventos, 94,7\% afirmaram que ocorreram na unidade de internação (enfermaria). Dos profissionais que responderam acerca da necessidade de afastamento do serviço em decorrência do episódio de agressão física, 14,9\% relataram afastamento do serviço por meio de licença médica.
Quanto ao registro da ocorrência de agressão por meio da Comunicação de Acidente de Trabalho (CAT), 54 profissionais agredidos relataram ter registrado o impresso (Tabela 2). No entanto, o número de casos de agressão registrados no Serviço de Segurança do Trabalho (SST) no período, por meio da CAT, foi de 37 (25 no primeiro hospital e 12 no outro), o que evidencia a discrepância entre o que estes profissionais responderam no questionário autoaplicável e o registro realizado no SST. No que diz respeito aos registros das ocorrências, o primeiro hospital apresentou acompanhamento mais completo quando comparado ao segundo, sendo colhidas as seguintes informações adicionais: diagnóstico principal do paciente, histórico de dependência química, tempo de internação, sexo do paciente agressor.

Tabela 2 Caracterização das ocorrências de agressão física contra profissionais de enfermagem de duas instituições psiquiátricas. Belo Horizonte, MG, Brasil, 2014

\begin{tabular}{lc}
\hline \multicolumn{1}{c}{ Variável } & $n(\%)$ \\
\hline Suporte da instituição $(\mathrm{n}=89)$ & $27(30,3)$ \\
$\quad$ Sim & $62(69,7)$ \\
Não & \\
Lesão física $(\mathrm{n}=88)$ & $25(28,4)$ \\
$\quad$ Sim & $63(71,6)$ \\
Não & \\
Consequências psicológicas $(\mathrm{n}=88)$ & $83(94,3)$ \\
Sim & $5(5,7)$ \\
Não & \\
Local onde ocorreu a agressão $(\mathrm{n}=94)$ & $89(94,7)$ \\
Enfermaria & $2(2,1)$ \\
Urgência & $3(3,2)$ \\
Pátio externo & \\
Registro da CAT $(\mathrm{n}=85)$ & $54(63,5)$ \\
Sim & $31(36,5)$ \\
Não & \\
Afastamento por licença médica $(\mathrm{n}=87)$ & $13(14,9)$ \\
Sim & $74(85,1)$ \\
Não &
\end{tabular}


Ao realizar a análise univariada (Tabela 3), não foi identificada relação estatística significante entre a ocorrência de agressão física e a variável turno de trabalho. Todavia, houve relação significante no que diz respeito ao sexo, pois as mulheres foram agredidas com maior frequência quando comparadas aos homens ( $\mathrm{p}$ $=0,039$ ). Também houve relação estatisticamente significante com a sensação de insegurança no ambiente de trabalho por parte dos profissionais de enfermagem $(p=0,008)$. O tempo de trabalho em instituições psiquiátricas também apresentou associação com a ocorrência de agressão física contra os profissionais de enfermagem, sendo que aqueles que relataram terem sido agredidos apresentavam maior tempo de trabalho (mais de 5 anos) nessas instituições ( $p=0,023$ ).

$\mathrm{Na}$ análise de regressão logística multivariada (Tabela 3), as variáveis sexo, percepção de segurança e tempo de trabalho em instituições psiquiátricas mantiveram significância estatística. Nessa análise, observou-se que as mulheres apresentaram 2,8 vezes mais chance de passar pela experiência de ser agredida fisicamente quando comparadas aos homens. Da mesma forma, os profissionais que relataram não se sentir seguros apresentavam 4,89 vezes mais chance de terem sido agredidos quando comparados àqueles que afirmaram se sentir seguros no hospital em que trabalhavam. Já os profissionais com tempo de experiência de trabalho em instituições psiquiátricas acima de cinco anos apresentaram 11,3 vezes mais chance de terem sido agredidos quando comparados àqueles que possuíam tempo de experiência menor ou igual a esse período.

Em relação aos pacientes que causaram agressões, considerando os 25 casos registrados em CATs no primeiro hospital, observou-se que $13(52,0 \%)$ eram do sexo masculino. Em 6 desses 25 casos, os pacientes envolvidos estiveram internados por mais de 95 dias, sendo a média de tempo de internação de 62,5 dias $(\mathrm{DP}=85,7$ dias $)$. No que diz respeito à dependência química, 20 (80,0\%) apresentaram histórico de uso de álcool ou outras drogas, sendo que 13 tiveram diagnóstico primário à internação relacionado ao uso de substâncias químicas e 7 à esquizofrenia.

\section{Discussão}

Esta pesquisa revelou o quanto é frequente os profissionais de enfermagem vivenciarem episódios de agressão física. Além disso, grande parte relatou sentimento de insegurança no ambiente de trabalho. Entre os profissionais que relataram ter passado pela experiência de algum tipo de agressão física, poucos afirmaram ter recebido suporte da instituição, apesar das consequências físicas e psicológicas relatadas. A despeito de ser comum a ocorrência desses eventos nesses dois hospitais psiquiátricos, o registro dos casos ainda é muito inferior aos relatos, não refletindo a real situação dentro dessas instituições.

Tabela 3 Análise de regressão logística univariada e múltipla acerca da associação entre a ocorrência de agressão física e as variáveis independentes turno de trabalho, sexo, percepção de segurança, tempo de trabalho em instituições psiquiátricas e tempo de vínculo com a instituição. Belo Horizonte, MG, Brasil, 2014

\begin{tabular}{|c|c|c|c|c|c|}
\hline & Variável & OR Univariada (IC95\%) & p-valor & OR Múltipla (IC95\%) & p-valor \\
\hline \multicolumn{6}{|c|}{ Turno $(n=125)$} \\
\hline Diurno & & 1 & \multirow{2}{*}{0,755} & & \\
\hline Noturno & & $0,866(0,352-2,131)$ & & & \\
\hline \multicolumn{6}{|l|}{$\operatorname{Sexo}(n=125)$} \\
\hline Masculino & & 1 & \multirow{2}{*}{0,039} & 1 & \multirow{2}{*}{0,039} \\
\hline Feminino & & $2,615(1,050-6,511)$ & & $2,803(1,056-7,443)$ & \\
\hline \multicolumn{6}{|c|}{ Sentimento de segurança no trabalho $(n=120)$} \\
\hline Sim & & 1 & \multirow{2}{*}{0,008} & 1 & \multirow{2}{*}{0,012} \\
\hline Não & & $4,339(1,465-12,855)$ & & $4,896(1,367-17,532)$ & \\
\hline \multicolumn{6}{|c|}{ Tempo de trabalho em instituições psiquiátricas ( $n=125)$} \\
\hline 0 a 5 anos & & 1 & \multirow{2}{*}{0,023} & 1 & \multirow{2}{*}{0,011} \\
\hline$>5$ anos & & $3,720(1,256-11,565)$ & & $11,331(1,745-37,558)$ & \\
\hline \multicolumn{6}{|c|}{ Tempo de vínculo (n = 125) } \\
\hline 0 a 5 anos & & 1 & \multirow{2}{*}{0,187} & 1 & \multirow{2}{*}{0,388} \\
\hline$>5$ anos & & $1,880(0,734-4,838)$ & & $0,516(0,115-2,313)$ & \\
\hline
\end{tabular}

OR= Odds Ratio; IC95\% = Intervalo de confiança de $95 \%$.

Estatística de teste de hipótese: utilizou-se análise de regressão logística univariada e multivariada. Para verificar o ajuste do modelo, utilizou-se o Teste de Hosmer-Lemeshow $=0,96 ; X^{2}=36,19$. 
A maior parte dos casos de agressão física ocorreu nas enfermarias, que são setores fechados para o trânsito livre dos pacientes internados e onde passam a maior parte do tempo. A literatura sugere que a restrição do espaço e a imposição de restrições aos pacientes exacerba os problemas relacionados à violência dentro dessas instituições ${ }^{7,14}$.

As consequências físicas e psicológicas relatadas pelos profissionais são reforçadas por outros estudos, que demonstraram a presença de transtorno do estresse pós-traumático e lesões físicas leves a severas, como lacerações de pele ou fratura ${ }^{2,3,11}$. As repercussões desses eventos impactam diretamente a saúde do profissional, levando ao adoecimento, medo, insatisfação e altas taxas de absenteísmo ${ }^{4,13}$.

As taxas encontradas neste estudo podem ser comparadas, com certa parcimônia, aos estudos realizados em outros países ${ }^{9,10,14,15}$. As diferentes classificações para as possíveis formas de violência devem ser consideradas, uma vez que os estudos utilizam diversas terminologias. Por exemplo, muitos abordam todas as formas de violência considerando a agressão física, verbal, sexual e psicológica. Além disso, existem diferenças metodológicas no que diz respeito aos tipos de estudo realizados, diferentes períodos de seguimento e formas de amostragem. Adicionalmente, a formação dos profissionais de saúde, a organização dos cuidados e o perfil dos pacientes atendidos podem apresentar diferenças capazes de influenciar a incidência desses eventos.

Dessa maneira, algumas estratégias são recomendadas para promover um ambiente de trabalho seguro, como treinamentos específicos para prevenir a violência, existência de políticas e procedimentos para promoção da segurança no ambiente hospitalar, identificação dos fatores que aumentam o risco de agressão, métodos de abordagem verbal para evitar o comportamento agressivo e uso de técnicas de autodefesa para os casos em que a abordagem verbal não for suficiente ${ }^{19}$.

Cabe ressaltar que a rotatividade dos profissionais entre os setores de hospitais psiquiátricos foi relacionada ao aumento da predisposição para episódios de agressão física, uma vez que os pacientes se sentem inseguros na presença de profissionais desconhecidos ${ }^{14}$. Além disso, estudo realizado anteriormente em um dos hospitais analisados nesta pesquisa demonstrou que os processos de comunicação são afetados com a rotatividade dos técnicos de enfermagem, limitando a gestão coletiva do risco de agressão física ${ }^{18}$.

A falta de suporte por parte da instituição, relatada pelos profissionais, e de registro desses eventos por meio das CATs, pode estar relacionada à percepção de que essas ocorrências são comuns e esperadas em hospitais psiquiátricos ${ }^{16}$. Além disso, esses incidentes geralmente só são notificados quando considerados graves pelos profissionais, o que acaba por camuflar o problema ${ }^{6}$.

No que se refere aos fatores associados a episódios de agressão física contra os técnicos de enfermagem, observou-se que o sexo do profissional, a percepção de segurança no ambiente de trabalho e o tempo de trabalho em instituições psiquiátricas apresentaram associação com episódios de violência física.

Os profissionais do sexo feminino mostraram-se mais vulneráveis quanto ao risco de ser agredido fisicamente nessas instituições, sendo este resultado confirmado por outros estudos ${ }^{1,12}$. No entanto, devem ser considerados os múltiplos fatores que predispõem a ocorrência de episódios de violência dentro de hospitais psiquiátricos, sejam eles aspectos relacionados à cultura organizacional, ao perfil de liderança, às atitudes dos profissionais, às características do ambiente e dos pacientes ${ }^{14,15}$.

Os profissionais da saúde tendem a se sentir inseguros em ambientes onde vivenciam com frequência episódios de agressão física. A literatura demonstra, por sua vez, que os profissionais de enfermagem tendem a enxergar as experiências de agressão física de forma altamente negativa, levando-os a desenvolver atitudes desfavoráveis no processo de cuidado aos pacientes, o que predispõem à ocorrência de novos episódios de agressão ${ }^{17}$. Dessa maneira, acredita-se que a percepção de segurança dentro do ambiente de trabalho não seja fator de risco para a ocorrência de episódios de agressão física, e sim uma variável proxy dos níveis de satisfação relacionados ao ambiente de trabalho e das atitudes dos profissionais em relação aos pacientes.

Discute-se que os fatores demográficos podem não apontar um perfil profissional específico que venha a predizer o risco de envolvimento em situações de violência, ressaltando que podem ser mais importantes as atitudes dos profissionais, competências, estilos de interação, habilidades de comunicação, fatores psicológicos e emocionais. Atitudes dos profissionais de saúde caracterizadas pela escuta qualificada, respeito e empatia em relação ao sofrimento do paciente, fornecimento de tratamento digno e presença de habilidades de comunicação/ negociação foram associadas com a redução de casos de violência ${ }^{14}$.

Profissionais que experimentam algum tipo de agressão física no local de trabalho tendem a se sentir mais inseguros e insatisfeitos neste ambiente e a desenvolver atitudes psicológicas desfavoráveis em relação aos pacientes. Essas atitudes, por sua vez, podem influenciar a qualidade da interação entre 
esses profissionais e os pacientes precipitando novos episódios de agressão física no ambiente de trabalho. Destaca-se que a percepção de segurança se encontra relacionada aos níveis de satisfação desses trabalhadores em relação às suas funções ${ }^{20}$. Baixos níveis de satisfação podem levar os profissionais de saúde a adotar atitudes negativas em relação ao paciente e, assim, predispor à experiência de ser agredido fisicamente no ambiente de trabalho ${ }^{14}$. Dessa forma, observa-se um ciclo entre ter passado pela experiência de agressão física, a qual influencia a percepção de segurança e a satisfação no ambiente de trabalho, que podem interferir nas atitudes desses profissionais e, consequentemente, nas chances de ocorrência de novos episódios de agressão física.

Apesar de outras pesquisas terem encontrado associação entre maior tempo de experiência profissional e menor chance de passar pela experiência de ser agredido fisicamente ${ }^{8,14}$, este estudo encontrou relação inversa. Isso talvez possa ser explicado por: maior nível de estresse entre profissionais com maior tempo de experiência ${ }^{2}$; mudança recente no perfil dos pacientes, passando do portador de patologias tradicionais a um maior número de dependentes químicos, o que exige novas estratégias de abordagem para as quais os profissionais participantes podem não estar preparados ${ }^{13}$, ou menor tolerância a qualquer ato de agressão, o que precipita a ocorrência de novos eventos ${ }^{17}$.

Considerando que as atitudes dos técnicos de enfermagem e os padrões de interação que estabelecem com os pacientes podem favorecer ou não a ocorrência de episódios de agressão física, destaca-se a importância de instituir programas de atualização que considerem a aquisição de conhecimentos e o desenvolvimento de habilidades e atitudes para o manejo dos pacientes em risco de heteroagressividade ou em situação de crise. Apesar de estudos anteriores apresentarem resultados conflitantes sobre a associação entre a redução da ocorrência de eventos agressivos dentro de enfermarias psiquiátricas e a realização de programas de atualização, ainda há muito a ser investigado ${ }^{14,16}$.

Deve-se ponderar que, de forma geral, os programas de atualização profissional ainda são idealizados e pautados na transmissão de conhecimento e no desenvolvimento de habilidades, não considerando a importância de estimular a mudança de atitudes em relação aos assuntos abordados ${ }^{21,22}$. Cumpre mencionar que programas de atualização que promovam a mudança de atitudes e, por conseguinte, dos comportamentos adotados pelos profissionais de saúde, devem ser estabelecidos com base em estratégias educativas que considerem os princípios da aprendizagem em adultos ${ }^{22}$. Ainda assim, esses comportamentos serão modificados e/ou mantidos em longo prazo apenas se existirem condições institucionais adequadas $^{23}$.

No que diz respeito ao perfil dos pacientes, a maior parte dos agressores era do sexo masculino e apresentou diagnóstico relacionado ao uso de álcool e outras drogas ou esquizofrenia. Além disso, foi observado que os casos registrados de agressão envolveram pacientes com maior tempo de internação. Apesar de não poder estabelecer qualquer associação com esse perfil, a definição das características desses pacientes pode contribuir para traçar perfis de risco dentro da instituição e, assim, estabelecer condutas preventivas para o gerenciamento do risco de agressão física.

Outros estudos demonstraram que existem algumas características dos pacientes associadas com episódios de violência, tais como idade, diagnóstico, sintomas e histórico prévio de comportamento violento ${ }^{14,15}$. Em relação ao diagnóstico, a história de dependência química, transtornos esquizoafetivos, esquizofrenia e quadros de mania foram identificados como um fator de risco para que o paciente apresente comportamentos de heteroagressividade. No que diz respeito à idade, os pacientes mais jovens possuem maior predisposição a apresentarem comportamentos violentos ${ }^{7,13-15}$.

Ressalta-se que organizações internacionais instituíram um conjunto de diretrizes para enfrentar o problema da violência no setor de saúde, visto que há tendências para o aumento de sua incidência, que impacta a qualidade de vida no trabalho e a saúde desses profissionais ${ }^{24}$. Nesse sentido, recomenda-se a adoção de programas e políticas formais direcionados à prevenção da violência dentro dessas instituições.

Por fim, medidas preventivas podem ser instituídas a fim de que melhorem as condições de trabalho para os profissionais de saúde e a qualidade da assistência aos pacientes atendidos ${ }^{18}$. Dentre as ações recomendadas, encontram-se o estabelecimento do mapeamento de risco dentro da instituição ao definir os pacientes com maior risco de envolvimento em episódios de violência, treinamento dos profissionais de saúde e suporte médico e psicológico aos que passam por essas experiências ${ }^{14}$.

Esta pesquisa apresenta algumas limitações relativas à natureza do estudo transversal, que impossibilitam encontrar as relações causais existentes. Adicionalmente, o questionário foi elaborado para ser autoaplicável, ficando sujeito à confiabilidade das respostas fornecidas pelos participantes. Os achados deste estudo são baseados em uma amostra autosselecionada e todos os resultados e interpretações são limitados a este grupo de respondentes. No entanto, destaca-se que se obteve uma taxa de 
resposta que representou praticamente a metade dos profissionais da instituição. Além disso, esta pesquisa foi realizada em dois dos quatro hospitais localizados no município. É importante mencionar que não foi possível analisar a influência dos cursos de capacitação profissional sobre a ocorrência de casos de agressão física contra os técnicos de enfermagem devido à ausência de dados fidedignos.

\section{Conclusão}

Os resultados permitiram observar que a ocorrência de agressão física, nos dois hospitais psiquiátricos, é vivenciada com frequência entre os técnicos de enfermagem. Constatou-se que grande parte dos casos de agressão física ocorreu nas enfermarias e no período diurno. A despeito das consequências físicas e psicológicas mencionadas pelos profissionais, chama-se atenção para o relato do pouco suporte da instituição e da presença de subnotificação dos eventos vivenciados por esses profissionais.

O maior número de profissionais do sexo feminino na enfermagem contribui para a ocorrência da violência ocupacional. Isso pôde ser evidenciado pelos resultados do estudo que demonstraram associação positiva entre a experiência de agressão física e ser do sexo feminino. Paralelamente, foi possível constatar um maior número de relatos relacionados à vivência de agressão física entre os profissionais com maior tempo de trabalho em instituições psiquiátricas e que relataram se sentir inseguros no ambiente de trabalho. Discute-se que as vivências prévias relacionadas à agressão física desencadeiam a percepção de insegurança no ambiente de trabalho, a insatisfação e o desenvolvimento de atitudes desfavoráveis frente aos pacientes, o que pode precipitar novos eventos.

Finalmente, os resultados apontam claramente para a necessidade de se instituir medidas que melhorem as condições de trabalho e que previnam a violência.

Sugere-se o desenvolvimento de estudos que visem investigar a influência dos cursos de capacitação profissional sobre a ocorrência de casos de agressão física contra os técnicos de enfermagem. Ainda, a realização de estudos que analisem as experiências vivenciadas por esses profissionais, uma vez que pesquisas de natureza qualitativa permitem compreender as questões que permeiam a prática dos profissionais e que não podem ser captadas em profundidade por estudos de abordagem quantitativa.

\section{Referências}

1. Zeng JY, An FR, Xiang YT, Qi YK, Ungvari GS, Newhouse R, et al. Frequency and risk factors of workplace violence on psychiatric nurses and its impact on their quality of life in China. Psychiatry Res. 2013;210(2):510-4.

2. Qi YK, Xiang YT, An FR, Wang J, Zeng JY, Ungvari GS, et al. Nurses' work-related stress in China: a comparison between psychiatric and general hospitals. Perspect Psychiatr Care. 2014;50(1):27-32.

3. Baby M, Glue P, Carlyle D. "Violence is not part of our job": a thematic analysis of psychiatric mental health nurses' experiences of patient assaults from a New Zealand perspective. Issues Ment Health Nurs. 2014;35(9):647-55.

4. Silva IV, Aquino EML, Pinto ICM. Violência no trabalho em saúde: a experiência de servidores estaduais da saúde no estado da Bahia, Brasil. Cad Saude Publica. 2014;30(10):2112-22.

5. Vasconcellos IRR, Abreu AMM, Maia EL. Violência ocupacional sofrida pelos profissionais de enfermagem do serviço de pronto atendimento hospitalar. Rev Gaúcha Enferm. 2012;33(2):16775.

6. Batista CB, Campus AS, Reis JC, Schall VT. Violência no trabalho em saúde: análise em unidades básicas de saúde de Belo Horizonte, Minas Gerais. Trab Educ Saúde. 2011;9(2):295-317.
7. Bowers L, Allan T, Simpson A, Jones J, Van Der Merwe M, Jeffery D. Identifying key factors associated with aggression on acute inpatient psychiatric wards. Issues Ment Health Nurs. 2009;30(4):260-71.

8. Kelly EL, Subica AM, Fulginiti A, Brekke JS, Novaco RW. A cross-sectional survey of factors related to inpatient assault of staff in a forensic psychiatric hospital. J Adv Nurs. 2015;71(5):1110-22.

9. Shiao JS, Tseng Y, Hsieh Y, Hou J, Cheng Y, Guo YL. Assaults against nurses of general and psychiatric hospitals in Taiwan. Int Arch Occup Environ Health. 2010;83(7):823-32.

10. Khoshknab MF, Tamizi Z, Ghazanfari N, Mehrabani G. Prevalence of workplace violence in psychiatric wards, Tehran, Iran. Pak J Biol Sci. 2012;15(14):680-4.

11. Moylan LB, Cullinan M. Frequency of assault and severity of injury of psychiatric nurses in relation to the nurses' decision to restrain. J Psychiatr Ment Health Nurs. 2011;18(6):526-34.

12. Chen W, Sun Y, Lan T, Chiu H. Incidence and risk factors of workplace violence on nursing staffs caring for chronic psychiatric patients in Taiwan. Int J Environ Res Public Health. 2009;6(11):2812-21. 
13. Broderick C, Azizian A, Kornbluh R, Warburton K. Prevalence of physical violence in a forensic psychiatric hospital system during 2011-2013: patients assaults, staff assaults and repeatedly violent patients. CNS Spectr. 2015;20(3):319-30.

14. Hamrin V, Iennaco J, Olsen D. A review of ecological factors affecting inpatient psychiatric unit violence: implications for relational and unit cultural improvements. Issues Ment Health Nurs. 2009;30(4):214-26.

15. Cornaggia CM, Beghi M, Pavone F, Barale F. Aggression in psychiatry wards: a systematic review. Psychiatry Res. 2011;189(1):10-20.

16. Stevenson KN, Jack SM, O’Mara L, LeGris J. Registered nurses' experiences of patient violence on acute care psychiatric inpatient units: an interpretative descriptive study. BMC Nurs. 2015;14:35.

17. James BO, Isa EW, Oud N. Patient aggression in psychiatric services: the experience of a sample of nurses at two psychiatric facilities in Nigeria. Afr J Psychiatry. 2011;14(1):130-3.

18. Mendes DP. Um enfoque sociológico sobre a gestão do risco de violência em hospitais públicos psiquiátricos: as dimensões do indivíduo. Trab Educ. 2012;21(1):129-42.

19. Peek-Asa C, Casteel C, Allareddy V, Nocera M, Goldmacher S, Ohagan E, et al. Workplace violence prevention programs in psychiatric units and facilities. Arch Psychiatr Nurs. 2009;23(2):166-76.

20. Vieira GLC, Mesquita TQO, Santos EO. Satisfação no trabalho entre técnicos de enfermagem em hospitais psiquiátricos de Minas Gerais - Brasil. Rev Min Enferm. 2015;19(1):167-73.

21. Tronchin DMR, Mira VL, Peduzzi M, Ciampone MHT, Melleiro MM, Silva JAM, et al. Educação permanente de profissionais de saúde em instituições públicas hospitalares. Rev Esc Enferm USP. 2009;43(Esp2):1210-5.

22. Johnson MJ, May CR. Promoting professional behavior change in healthcare: what interventions work, and why? A theory-led overview of systematic reviews. BMJ Open. 2015;5(9):e008592.

23. Fishbein M, Ajzen I. Predicting and changing behavior: the reasoned action approach. New York: Psychology Press; 2010.

24. Organización Internacional del Trabajo (OIT). Consejo Internacional de enfermeras (CIE). Organización Mundial de la Salud (OMS). Internacional de Servicios Públicos (ISP). Directrices marco para afrontar la violencia laboral en el sector de la salud. Genebra: OIT; 2002. Disponível em: http://www.ilo.org/wcmsp5/ groups/public/---ed_dialogue/---sector/documents/ publication/wcms_160911.pdf 